\title{
Sociodemographic characteristics and food habits of organic consumers - a study from the Danish National Birth Cohort
}

\author{
Sesilje B Petersen ${ }^{1, *}$, Morten A Rasmussen², Marin Strøm ${ }^{1}$, Thorhallur I Halldorsson ${ }^{1,3}$ \\ and Sjurdur F Olsen ${ }^{1,4}$ \\ ${ }^{1}$ Centre for Fetal Programming, Department of Epidemiology Research, Statens Serum Institut, Artillerivej 5, \\ Building 206, Room 306, DK-2300 Copenhagen S, Denmark: ${ }^{2}$ Department of Food Science/Quality and \\ Technology, Faculty of Life Sciences, Copenhagen, Denmark: ${ }^{3}$ Unit for Nutrition Research, Faculty of Food \\ Science and Nutrition, School of Health Sciences, University of Iceland, Reykjavik, Iceland: ${ }^{4}$ Department of \\ Nutrition, Harvard School of Public Health, Boston, MA, USA
}

Submitted 21 July 2011: Final revision received 22 June 2012: Accepted 5 July 2012: First published online 12 September 2012

\begin{abstract}
Objective: To develop a basis for building models that can examine the impact of organic food (OF) choices on maternal and offspring health, including identification of factors associated with $\mathrm{OF}$ consumption and underlying dietary patterns.

Design: Dietary intake was collected for the preceding month from an FFQ in mid-pregnancy and information on sociodemographic characteristics was collected from telephone interviews during pregnancy. From a question about OF consumption in the FFQ, including six food categories, an OF preference index was calculated. Latent variables that captured the variability in OF choices in relation to dietary intake were defined.

Setting: The Danish National Birth Cohort (DNBC), 1996-2002.

Subjects: Pregnant women from DNBC ( $n$ 60 773).

Results: We found that frequent OF use was highly associated with age, occupational status, urbanization, smoking and vegetarianism. By principal components analysis we identified two eating patterns, a 'Western dietary pattern' and a 'Prudent dietary pattern', that explained $14 \cdot 2 \%$ of the variability in data. Frequent OF users consumed a more 'prudent' diet compared with non-users and had significantly higher intakes of vegetables $(+67 \%)$, fibre $(+13 \%)$ and $n-3$ fatty acids $(+11 \%)$ and less saturated fat $(-8 \%)$.

Conclusions: Frequent OF users seemed to have a healthier lifestyle than non-users. These findings highlight a major challenge in observational studies examining the impact of OF consumption on health due to potentially irremediable confounding factors.
\end{abstract}

Keywords
Organic food
Maternal diet
Epidemiology
During the past decade the demand for organic food (OF) products has grown considerably and various alternatives to the mainstream conventional food products and distribution have been developed and are increasing, especially in developed countries ${ }^{(1)}$. It is difficult to identify reasons for this growth as studies show that OF consumption reflects a complex web of determinants including its availability in food stores, sociodemographic and cultural factors, and personal values and attitudes. Studies, however, also reveal that health considerations are a major factor behind the growth in OF consumption $^{(2-12)}$. This is noteworthy because a causal association between OF consumption and better health remains to be scientifically established ${ }^{(13-18)}$. Several types of study have been conducted to address the question, but with few or unclear answers for various reasons: the biological and chemical studies in the field are often incomparable, as chemical composition of crops is easily affected by temperature, soil and variety ${ }^{(5,7,8,14)}$; few comparable animal studies exist and some may not be relevant for today's farming practice ${ }^{(13,17)}$; and very few intervention studies or prospective observational studies have addressed the potential health benefits of OF products ${ }^{(16,19-21)}$.

In relation to the health effects of OF consumption, the observational study design is complicated by the role of potential confounding factors. Thus, underlying determinants of OF purchase need to be described and included in statistical models in order to minimize potential confounding. The Danish National Birth Cohort (DNBC) $)^{(22,23)}$ is suitable for addressing this issue as it is one of the largest prospective cohort studies worldwide to have recorded OF preferences during pregnancy along 
with a multitude of other dietary, health and socioeconomic factors. Furthermore, Denmark has a strong system for control and certification of organic farming and manufacturing, a matured organic market and the highest consumption of OF per capita in Europe ${ }^{(24)}$. Therefore, the DNBC offers unique opportunities to study the impact of OF in pregnancy on maternal and offspring health.

The aim of the present study was to develop a basis for building models that can examine the impact of $\mathrm{OF}$ choices on maternal and offspring health among Danish pregnant women. The first step was to identify factors associated with OF consumption. The second step was to employ multivariate methods to identify underlying patterns and to define latent variables that can capture the variability in OF choices among the study population.

\section{Materials and methods}

\section{The Danish National Birth Cobort}

The DNBC is a cohort with information from 100000 pregnancies $^{(22)}$. Women were recruited between 1996 and 2002 during the first antenatal visit to the general practitioner at around weeks $6-10$ of gestation. The data collection in the study included four telephone interviews (two prenatal interviews conducted in gestational weeks 12 and 30, and two postnatal interviews when the child was 6 and 18 months old) and a semi-quantitative FFQ mailed to the women in week 25 of gestation ${ }^{(22,23)}$. It was estimated that during the study period approximately $35 \%$ of all deliveries in Denmark were included in the cohort $^{(22)}$. Some of the women are registered in the cohort twice or more through subsequent pregnancies during the recruitment period. However, in our study, only the first pregnancy for each participating woman was included to avoid inter-correlation within subjects. Furthermore, multiple pregnancies were excluded from the study. The DNBC complies with the Declaration of Helsinki and was approved by the Danish National Committee on Biomedical Research Ethics.

\section{Definition of organic food preference index}

Information on OF consumption was collected through the $\mathrm{FFQ}^{(23)}$. About $70 \%$ of the women returned the questionnaire, which was a modified form of a questionnaire used by the Danish Cancer Registry ${ }^{(25)}$. Of the 100000 women in DNBC, 60773 met the inclusion criteria and had answered the question about OF consumption in the FFQ.

The FFQ covered the pregnant woman's diet during the preceding 4 weeks and included one question about $\mathrm{OF}$ consumption: 'How often do you eat organic foods?'. The question was divided into six categories: 'milk products', 'cereals', 'egg', 'vegetables', 'fruit' and 'meat'. The answer categories were 'never', 'sometimes', 'regularly' or 'always'. Based on the question we calculated an OF preference index. Each answer category was given a score (never $=1$, sometimes $=2$, regularly $=3$, always $=4$ ) and summarized across categories to form an organic index (OX). Missing values were characterized as 'never eating organic'. Due to the fact that vegetarians and vegans in general have no consumption of meat and vegans have no consumption of meat, egg and milk products, the meat category was excluded for both groups and the egg and milk categories were excluded for vegans. Based on the constructed index the women were divided into four groups: 'non-users' $(\mathrm{OX}=6)$, 'low users' $(6<\mathrm{OX} \leq 12)$, 'moderate users' $(12<\mathrm{OX} \geq 18)$ and 'frequent users' $(\mathrm{OX}>18)$.

\section{Assessment of dietary patterns}

In the FFQ the women were asked about frequencies for approximately 360 different food and beverage items. To estimate food intake standard portion sizes and standard recipes were applied for all items in the questionnaire. Standard portion sizes were multiplied with the daily frequencies to estimate the intake of each food item in grams. For more complex items standard recipes were made and the intakes of foods present in different items were aggregated. The estimated amounts of all items were coupled with the Danish food composition tables ${ }^{(26)}$ and corrected for loss of fat, water, vitamins and minerals. The 360 different items were divided into thirty-five main food groups and sixty-five more specific food groups representing the entire diet of the women. All nutrients were energy adjusted by the residual method, as described by Willett et $a l^{(27)}$. Only women with an energy intake $>4500 \mathrm{~kJ} / \mathrm{d}$ and $<20000 \mathrm{~kJ} / \mathrm{d}$ were included in the analyses for dietary intakes to avoid unrealistic estimates. The FFQ used in the DNBC has been validated in a group of younger non-pregnant women ${ }^{(28)}$ and in the DNBC for the intake of fruit, vegetables and pregnancy-relevant nutrients (folate, protein, retinol and $n-3$ fatty acids) by a $7 \mathrm{~d}$ weighed food diary and biomarkers ${ }^{(29,30)}$.

\section{Assessment of sociodemographic and lifestyle factors}

Sociodemographic and lifestyle variables were gathered from the consent form, the FFQ and the telephone interviews and included: age $(<20,20-24,25-29,30-34$, 35-39, $\geq 40$ years); parity (0, 1, 2, 3+); occupational status (high-level proficiencies, medium-level proficiencies, skilled, student, unskilled, unemployed); cohabitation status (single, couple/married); urbanization (capital city, capital suburbs, $100000+, 10000-99999,<10000$ citizens); smoking during pregnancy (non-smoker, occasional smoker, $<15$ cigarettes/d, $\geq 15$ cigarettes/d); alcohol intake in pregnancy (not at all, yes); energy intake (in quintiles); physical activity (none, light, moderate, high level); intake of dietary supplements in the pregnancy (no, yes); maternal pre-pregnant BMI $(<18 \cdot 5$, $\left.18 \cdot 5-24 \cdot 9, \quad 25 \cdot 0-29 \cdot 9, \quad 30 \cdot 0-34 \cdot 9, \geq 35 \cdot 0 \mathrm{~kg} / \mathrm{m}^{2}\right)$; living area in Denmark (West or East part of the country); and vegetarianism (yes, no). Most of these variables have 
been described and used in earlier studies based on the $\mathrm{DNBC}^{(31-40)}$.

\section{Statistical analysis}

Univariate and multivariate logistic regression was used to estimate the association between OF consumption and sociodemographic characteristics and linear regression was used to analyse differences in dietary intake between non-users and frequent OF users. In these analyses the focus was on the differences between non-users and frequent users in order to obtain the biggest contrast between OF consumers. All analyses were performed with the SAS statistical software package version $9 \cdot 1$.

Principal components analysis (PCA) was used for exploration of the associations between the sixty-five food groups. PCA is conducted to uncover the systematic correlation structure between variables while excluding the non-systematic variation. PCA is a widely used method for compression of large data sets, often with a large number of variables, into a few underlying latent variables (principal components), describing the systematic variation. The number of variables in the present work is manageable from a univariate point of view, and PCA is hence not applied as a 'second to none' alternative compared with the univariate analysis. PCA in combination with visualization reveals the inter-variable correlation structure and hence adds a dimension on top of what can be explored from univariate analysis ${ }^{(41)}$. PCA models were implemented in MATLAB version $7 \cdot 9 \cdot 0 \cdot 529$ (R2009b) using PLS toolbox version $5 \cdot 2 \cdot 2$ (Eigenvector Research Inc.) and in-house algorithms for plotting of results. The Spearman rank correlation test was used to find the correlation between principal components and OF consumption.

\section{Results}

The responses to the OF question are shown in Table 1. The frequencies of organic consumption through the six food categories differed substantially. The consumption of organic eggs and milk was common among the women, whereas intakes of organic cereals, vegetables, fruit and meat were low. According to the constructed OF preference index, $12 \%$ of the study population were classified as non-users, $44 \%$ were low users, $37 \%$ moderate users and $7 \%$ were frequent users.

Table 2 shows associations between OF preferences and sociodemographic and lifestyle characteristics as odds ratios and 95\% confidence intervals for being a frequent user as opposed to a non-user. The crude OR are based on univariate analyses, whereas the adjusted OR are based on a multivariate analysis showing the associations with OF preferences for each explaining variable independently of all the other variables in the table. The
Table 1 Distribution of answers in the six food categories: pregnant women (n 60773), Danish National Birth Cohort (DNBC), 1996-2002

\begin{tabular}{|c|c|c|c|c|c|c|c|c|}
\hline \multirow{2}{*}{$\begin{array}{l}\text { Organic } \\
\text { food }\end{array}$} & \multicolumn{2}{|c|}{ Never } & \multicolumn{2}{|c|}{ Sometimes } & \multicolumn{2}{|c|}{ Regularly } & \multicolumn{2}{|c|}{ Always } \\
\hline & $n$ & $\%$ & $n$ & $\%$ & $n$ & $\%$ & $n$ & $\%$ \\
\hline Milk & 15865 & 26 & 20679 & 34 & 16366 & 27 & 7862 & 13 \\
\hline Cerea & 18666 & 31 & 26482 & 43 & 12664 & 21 & 2961 & 5 \\
\hline Egg & 15825 & 26 & 174 & 29 & 115 & 19 & 15958 & 26 \\
\hline Vegetables & 15162 & 25 & 310 & 51 & 13065 & 22 & 1723 & 2 \\
\hline Fruit & 24994 & 36 & 35089 & 50 & 8630 & 12 & 1464 & 2 \\
\hline Meat & 31107 & 52 & 23468 & 39 & 4675 & 8 & 820 & 1 \\
\hline
\end{tabular}

women's age had a strong and independent association with OF preferences and so did vegetarianism. Social group, smoking, BMI, physical activity, living area and urbanization were all associated with OF preferences in the crude analysis; however, adjusting for other factors attenuated their associations with OF preferences, although living area and urbanization still had strong associations. Regarding cohabitation status, adjustment tended to strengthen the association with OF preferences. For alcohol intake, use of dietary supplements, physical activity and occupational status the association with OF preferences was eliminated upon adjustment for the other covariates.

Table 3 shows the mean daily intakes of food items for non-users and the adjusted increments in intake for frequent users compared with non-users. Intakes differed significantly across OF preference for almost all foods and food groups. The most marked differences in intake were observed for vegetables, legumes, fruit and berries, nuts, lamb, seafood, plant oils and tea; all with higher intakes for frequent $\mathrm{OF}$ consumers. Adjustment for covariates attenuated the observed differences and reversed the association between OF preferences and the intake of alcohol and desserts (candy, ice cream and cakes). Compared with non-users, frequent users seemed to substitute certain items with others, e.g. margarines with oils, white bread with dark bread, pork with poultry, lamb with fish, coffee with tea and soft drinks with water and juice.

Table 4 shows daily intakes of specified nutrients according to OF preferences. Nearly all comparisons between frequent users and non-users were statistically significant. The most marked differences were observed for $n$-3 fatty acids, fibre, iodine, $\beta$-carotene, folate and vitamins $\mathrm{D}, \mathrm{K}$ and $\mathrm{C}-$ which were higher among frequent users - and SFA, MUFA, n-6 fatty acids, trans fatty acids, cholesterol and retinol - which were lower among frequent users. Adjustment attenuated the differences; however, significantly higher intakes of certain nutrients were still observed.

Figure 1 shows results from the PCA. Inter-correlation of food groups is shown as a scatter plot of the first two principal components for the food groups. The food 
Table 2 Association between organic food preferences and different sociodemographic factors (odds ratios for being a frequent user as opposed to a non-user): pregnant women ( $n$ 60773), Danish National Birth Cohort (DNBC), 1996-2002

\begin{tabular}{|c|c|c|c|c|c|c|c|c|c|}
\hline \multirow[b]{2}{*}{ Variable } & \multicolumn{2}{|c|}{ Non-users } & \multicolumn{2}{|c|}{ Frequent users } & \multicolumn{2}{|c|}{ Crude } & \multicolumn{2}{|c|}{ Adjusted } & \multirow[b]{2}{*}{$P+$} \\
\hline & $n$ & $\%$ & $n$ & $\%$ & OR & $95 \% \mathrm{Cl}^{*}$ & $\mathrm{OR}^{*}$ & $95 \% \mathrm{Cl}^{*}$ & \\
\hline Age (years) & & & & & & & & & $<0.0001$ \\
\hline$<20$ & 148 & 2 & 29 & 1 & 0.43 & $0.29,0.65$ & 0.33 & $0 \cdot 16,0 \cdot 70$ & \\
\hline $20-23$ & 1476 & 21 & 296 & 7 & 0.44 & $0.39,0.51$ & 0.48 & $0.39,0.60$ & \\
\hline $25-29$ & 3102 & 43 & 1402 & 34 & Ref. & - & Ref. & - & \\
\hline 30-34 & 1901 & 26 & 1646 & 40 & 1.92 & $1 \cdot 75,2 \cdot 10$ & $1 \cdot 88$ & $1 \cdot 64,2 \cdot 16$ & \\
\hline $35-39$ & 491 & 7 & 692 & 17 & $3 \cdot 12$ & $2 \cdot 73,3 \cdot 56$ & $3 \cdot 56$ & $2 \cdot 90,4 \cdot 37$ & \\
\hline$\geq 40$ & 47 & 1 & 81 & 2 & $3 \cdot 81$ & $2 \cdot 65,5 \cdot 49$ & $4 \cdot 49$ & $2 \cdot 71,7 \cdot 45$ & \\
\hline Occupational status & & & & & & & & & $<0.0001$ \\
\hline High-level proficiencies & 266 & 4 & 519 & 14 & Ref. & - & Ref. & - & \\
\hline Medium-level proficiencies & 1107 & 17 & 1264 & 33 & 0.58 & $0.49,0.69$ & 0.83 & $0.67,1.04$ & \\
\hline Skilled & 1534 & 23 & 484 & 13 & $0 \cdot 16$ & $0 \cdot 13,0 \cdot 19$ & 0.27 & $0.21,0.34$ & \\
\hline Student & 543 & 8 & 654 & 17 & 0.62 & $0.51,0.74$ & $1 \cdot 01$ & $0 \cdot 78,1 \cdot 31$ & \\
\hline Unskilled & 2194 & 33 & 447 & 12 & $0 \cdot 10$ & $0 \cdot 09,0 \cdot 12$ & 0.25 & $0 \cdot 20,0 \cdot 32$ & \\
\hline Unemployed & 984 & 15 & 432 & 11 & $0 \cdot 22$ & $0 \cdot 19,0 \cdot 27$ & 0.48 & $0.37,0.62$ & \\
\hline Living area & & & & & & & & & $<0.0001$ \\
\hline West Denmark & 5212 & 77 & 1617 & 43 & Ref. & - & Ref. & - & \\
\hline East Denmark & 1567 & 23 & 2100 & 57 & $4 \cdot 32$ & $3 \cdot 96,4 \cdot 71$ & $2 \cdot 44$ & $2 \cdot 05,2 \cdot 90$ & \\
\hline Urbanization & & & & & & & & & $<0.0001$ \\
\hline Capital city & 216 & 4 & 1036 & 29 & $23 \cdot 8$ & $20 \cdot 1,28 \cdot 2$ & $8 \cdot 04$ & $6 \cdot 23,10 \cdot 4$ & \\
\hline Capital suburbs & 333 & 5 & 480 & 13 & $7 \cdot 15$ & $6 \cdot 07,8 \cdot 44$ & $2 \cdot 70$ & $2 \cdot 10,3 \cdot 48$ & \\
\hline $100000+$ & 590 & 9 & 673 & 19 & $5 \cdot 66$ & $4 \cdot 92,6 \cdot 52$ & $5 \cdot 04$ & $4 \cdot 22,6 \cdot 02$ & \\
\hline $10000-99999$ & 2124 & 34 & 801 & 22 & $1 \cdot 87$ & $1 \cdot 66,2 \cdot 11$ & $1 \cdot 45$ & $1 \cdot 25,1 \cdot 69$ & \\
\hline$<10000$ & 3988 & 48 & 602 & 17 & Ref. & - & Ref. & - & \\
\hline Cohabitation status & & & & & & & & & 0.0001 \\
\hline Couple/married & 6731 & 98 & 3831 & 97 & Ref. & - & Ref. & - & \\
\hline Single & 125 & 2 & 103 & 3 & $1 \cdot 45$ & $1 \cdot 11,1 \cdot 88$ & $2 \cdot 25$ & $1 \cdot 43,3 \cdot 55$ & \\
\hline Parity & & & & & & & & & $0 \cdot 66$ \\
\hline 0 & 3649 & 53 & 1789 & 45 & Ref. & - & Ref. & - & \\
\hline 1 & 1990 & 29 & 1520 & 39 & $1 \cdot 56$ & $1 \cdot 46,1 \cdot 70$ & $1 \cdot 54$ & $1 \cdot 34,1 \cdot 76$ & \\
\hline 2 & 941 & 14 & 537 & 14 & $1 \cdot 16$ & $1 \cdot 03,1 \cdot 31$ & $1 \cdot 15$ & $0.95,1.39$ & \\
\hline $3+$ & 278 & 4 & 89 & 2 & 0.65 & $0.51,0.83$ & 0.43 & $0.29,0.64$ & \\
\hline Smoking & & & & & & & & & $<0.0001$ \\
\hline Non-smoker & 5014 & 71 & 3210 & 78 & Ref. & - & Ref. & - & \\
\hline Occasional smoker & 741 & 10 & 559 & 14 & $1 \cdot 18$ & $1 \cdot 05,1 \cdot 33$ & $1 \cdot 34$ & $1 \cdot 12,1 \cdot 61$ & \\
\hline$<15$ cigarettes/d & 1091 & 15 & 313 & 8 & 0.45 & $0.39,0.51$ & 0.62 & $0.50,0 \cdot 75$ & \\
\hline$\geq 15$ cigarettes $/ d$ & 264 & 4 & 33 & 1 & $0 \cdot 20$ & $0 \cdot 14,0.28$ & $0 \cdot 28$ & $0 \cdot 16,0 \cdot 48$ & \\
\hline Alcohol in pregnancy & & & & & & & & & $0 \cdot 21$ \\
\hline Not at all & 3506 & 49 & 1803 & 44 & Ref. & - & Ref. & - & \\
\hline Yes & 3600 & 51 & 2309 & 56 & $1 \cdot 25$ & $1 \cdot 15,1 \cdot 35$ & $0 \cdot 90$ & $0 \cdot 80,1 \cdot 01$ & \\
\hline Dietary habits & & & & & & & & & $<0.0001$ \\
\hline Eating meat & 7172 & $99 \cdot 7$ & 3909 & 94 & Ref. & - & Ref. & - & \\
\hline Vegetarian/vegan & 23 & 0.3 & 237 & 6 & $18 \cdot 8$ & $12 \cdot 2,28 \cdot 9$ & $18 \cdot 7$ & $9 \cdot 77,35 \cdot 7$ & \\
\hline Pre-pregnant BMI $\left(\mathrm{kg} / \mathrm{m}^{2}\right)$ & & & & & & & & & $<0.0001$ \\
\hline$<18.5$ & 274 & 4 & 229 & 6 & $1 \cdot 15$ & $0 \cdot 96,1 \cdot 38$ & $1 \cdot 26$ & $0 \cdot 96,1 \cdot 66$ & \\
\hline $18 \cdot 5-24 \cdot 9$ & 4016 & 60 & 2911 & 75 & Ref. & - & Ref. & - & \\
\hline $25 \cdot 0-29 \cdot 9$ & 1604 & 24 & 561 & 15 & 0.48 & $0.43,0.54$ & 0.68 & $0.58,0.79$ & \\
\hline $30 \cdot 0-34 \cdot 9$ & 594 & 9 & 127 & 3 & 0.29 & $0.24,0.36$ & 0.51 & $0.39,0.67$ & \\
\hline$\geq 35 \cdot 0$ & 252 & 4 & 39 & 1 & $0 \cdot 21$ & $0 \cdot 15,0 \cdot 30$ & $0 \cdot 31$ & $0.19,0.51$ & \\
\hline Energy intake & & & & & & & & & $<0.0001$ \\
\hline Quintile 1 & 1573 & 22 & 668 & 16 & Ref. & - & Ref. & - & \\
\hline Quintile 2 & 1290 & 19 & 762 & 19 & $1 \cdot 39$ & $1 \cdot 22,1 \cdot 58$ & $1 \cdot 23$ & $1 \cdot 02,1 \cdot 49$ & \\
\hline Quintile 3 & 1342 & 19 & 790 & 19 & $1 \cdot 38$ & $1 \cdot 22,1 \cdot 57$ & $1 \cdot 24$ & $1 \cdot 03,1 \cdot 50$ & \\
\hline Quintile 4 & 1270 & 18 & 889 & 22 & $1 \cdot 65$ & $1 \cdot 45,1 \cdot 87$ & 1.59 & $1 \cdot 32,1 \cdot 91$ & \\
\hline Quintile 5 & 1509 & 22 & 980 & 24 & 1.53 & $1 \cdot 35,1 \cdot 73$ & $1 \cdot 80$ & $1 \cdot 50,-2 \cdot 15$ & \\
\hline Physical activity & & & & & & & & & $<0.0001$ \\
\hline None & 4673 & 68 & 2162 & 55 & Ref. & - & Ref. & - & \\
\hline Light & 1358 & 20 & 910 & 23 & $1 \cdot 45$ & $1 \cdot 31,1 \cdot 60$ & $1 \cdot 30$ & $1 \cdot 12,1 \cdot 49$ & \\
\hline Moderate & 718 & 11 & 734 & 19 & $2 \cdot 21$ & $1 \cdot 97,2 \cdot 48$ & $1 \cdot 72$ & $1 \cdot 45,2 \cdot 03$ & \\
\hline High & 92 & 1 & 110 & 3 & $2 \cdot 58$ & $1.95,3.42$ & $1 \cdot 24$ & $0.80,1 \cdot 93$ & \\
\hline Dietary supplements & & & & & & & & & 0.073 \\
\hline No & 671 & 10 & 211 & 5 & Ref. & - & Ref. & - & \\
\hline Yes & 6334 & 90 & 3891 & 95 & $1 \cdot 95$ & $1 \cdot 66,2 \cdot 29$ & $1 \cdot 28$ & $1 \cdot 00,1 \cdot 64$ & \\
\hline
\end{tabular}

Ref, referent category.

${ }^{*}$ Mutually adjusted.

$+P$ value for trend for exposures with more than two categories. 
Table 3 Associations between the intake of different food items and organic consumption: pregnant women ( $n$ 60 773), Danish National Birth Cohort (DNBC), 1996-2002

\begin{tabular}{|c|c|c|c|c|c|}
\hline \multirow[b]{3}{*}{ Food item } & \multirow{2}{*}{\multicolumn{2}{|c|}{ Non-users }} & \multicolumn{3}{|c|}{ Frequent users* } \\
\hline & & & \multicolumn{3}{|c|}{ Increase in intaket } \\
\hline & Mean & SD & $g / d$ & $95 \% \mathrm{Cl}$ & $\%$ \\
\hline Vegetables & $92 \cdot 4$ & $82 \cdot 7$ & $61 \cdot 9$ & $58 \cdot 1,65 \cdot 8$ & 67 \\
\hline Legumes & $9 \cdot 5$ & $17 \cdot 6$ & $6 \cdot 2$ & $5 \cdot 3,7 \cdot 2$ & 65 \\
\hline Fruit and berries & 128 & 104 & $42 \cdot 0$ & $37 \cdot 3,46 \cdot 7$ & 33 \\
\hline Nuts & $1 \cdot 2$ & $2 \cdot 6$ & $1 \cdot 5$ & $1 \cdot 3,1 \cdot 7$ & 125 \\
\hline Potatoes & 144 & 103 & $-3 \cdot 7$ & $-7 \cdot 1,-2 \cdot 4$ & -3 \\
\hline French fries & $9 \cdot 4$ & $10 \cdot 9$ & $-2 \cdot 8$ & $-3 \cdot 1,-2 \cdot 4$ & -30 \\
\hline Rice & $10 \cdot 1$ & $8 \cdot 2$ & $1 \cdot 4$ & $1 \cdot 1,1 \cdot 8$ & 14 \\
\hline Pasta & $13 \cdot 0$ & $9 \cdot 5$ & $1 \cdot 3$ & $0 \cdot 9,1 \cdot 7$ & 10 \\
\hline Wholegrain bread/flour & 135 & 84 & $13 \cdot 0$ & $9 \cdot 8,16 \cdot 3$ & 10 \\
\hline White bread/flour & $93 \cdot 8$ & $58 \cdot 8$ & $-14 \cdot 1$ & $-16 \cdot 2,-12 \cdot 1$ & -15 \\
\hline Breakfast cereals & $25 \cdot 6$ & $28 \cdot 3$ & $6 \cdot 2$ & $5 \cdot 0,7 \cdot 5$ & 24 \\
\hline Poultry & $19 \cdot 7$ & $17 \cdot 8$ & $2 \cdot 6$ & $1 \cdot 8,3 \cdot 5$ & 13 \\
\hline Pork & $29 \cdot 9$ & $19 \cdot 9$ & -8.9 & $-9 \cdot 6,-8 \cdot 3$ & -30 \\
\hline Beef/veal & $41 \cdot 7$ & $30 \cdot 7$ & $1 \cdot 1$ & $0 \cdot 1,2 \cdot 2$ & 3 \\
\hline Lamb & $0 \cdot 8$ & $4 \cdot 1$ & $2 \cdot 1$ & $1 \cdot 9,2 \cdot 3$ & 263 \\
\hline Processed meat & $19 \cdot 5$ & $15 \cdot 4$ & $-5 \cdot 3$ & $-5 \cdot 8,-4 \cdot 8$ & -27 \\
\hline Seafood & $22 \cdot 7$ & $28 \cdot 0$ & $9 \cdot 3$ & $8 \cdot 4,10 \cdot 2$ & 41 \\
\hline Egg & $14 \cdot 7$ & $14 \cdot 1$ & 1.5 & $1 \cdot 1,2 \cdot 0$ & 10 \\
\hline Whole-fat milk products & $67 \cdot 2$ & 175 & $-8 \cdot 8$ & $-14 \cdot 4,-3 \cdot 1$ & -13 \\
\hline Light milk products & 478 & 407 & $18 \cdot 9$ & $2 \cdot 2,35 \cdot 7$ & 4 \\
\hline Yoghurt & $43 \cdot 2$ & $56 \cdot 0$ & $8 \cdot 1$ & $5 \cdot 7,10 \cdot 6$ & 19 \\
\hline Butter & $7 \cdot 7$ & $9 \cdot 3$ & $1 \cdot 5$ & $1 \cdot 1,1 \cdot 9$ & 20 \\
\hline Cheese & $28 \cdot 2$ & $24 \cdot 2$ & $4 \cdot 5$ & $3 \cdot 5,5 \cdot 6$ & 16 \\
\hline Oils & 0.9 & $2 \cdot 1$ & $1 \cdot 2$ & $1 \cdot 1,1 \cdot 3$ & 133 \\
\hline Margarine & $23 \cdot 2$ & $22 \cdot 0$ & $-5 \cdot 1$ & $-5 \cdot 9,-4 \cdot 4$ & -22 \\
\hline Dressing/sauce & $5 \cdot 6$ & $7 \cdot 9$ & $-1 \cdot 2$ & $-1 \cdot 5,-1 \cdot 0$ & -21 \\
\hline Tea & 122 & 202 & $33 \cdot 0$ & $23 \cdot 6,42 \cdot 4$ & 27 \\
\hline Coffee & 165 & 266 & $-32 \cdot 4$ & $-41 \cdot 8,-23 \cdot 0$ & -20 \\
\hline Drink, sweated & 203 & 300 & $-29 \cdot 0$ & $-41 \cdot 8,-16 \cdot 2$ & -14 \\
\hline Drink, light & 251 & 333 & $-30 \cdot 0$ & $-36 \cdot 5,-23 \cdot 4$ & -12 \\
\hline Juice & 167 & 268 & $20 \cdot 8$ & $10 \cdot 5,31 \cdot 0$ & 12 \\
\hline Water & 985 & 553 & 118 & $94 \cdot 9,141$ & 12 \\
\hline Alcohol & $20 \cdot 3$ & $37 \cdot 5$ & $-1 \cdot 6$ & $-3 \cdot 4,0 \cdot 3$ & -8 \\
\hline Snack & $4 \cdot 8$ & $5 \cdot 3$ & $-1 \cdot 4$ & $-1 \cdot 6,-1 \cdot 2$ & -29 \\
\hline Dessert & $43 \cdot 3$ & $31 \cdot 2$ & $-2 \cdot 8$ & $-3 \cdot 9,-1 \cdot 6$ & -6 \\
\hline
\end{tabular}

${ }^{*}$ Change compared with non-users.

tMultivariate linear regression. Covariates: cohabitation status, age, smoking habits, parity, pre-pregnant BMI, occupational status, physical activity, energy intake, urbanization and living area.

groups are coloured according to common food classes. When two variables are positioned close to each other they are correlated with respect to the variance explained by the two components.

From the PCA (Fig. 1) two distinct eating patterns, describing $14 \cdot 2 \%$ of the total variation in data, can be derived. PC1 is associated with a dietary pattern comprising more vegetables, cabbage, roots, legumes, fish, etc., as these food groups obtain high positive values in PC1. We named this component the 'prudent dietary pattern'. PC2 is characterized by a high intake of pork, mixed/processed meat, white bread, margarine, French fries, etc., as these food groups obtain high positive values in PC2. This component we named the 'Western dietary pattern'. The prudent dietary pattern was found to be positively correlated with frequent OF consumption $(r=0 \cdot 26, \quad P<0 \cdot 00001)$, whereas the Western dietary pattern was negatively correlated with $\mathrm{OF}$ consumption $(r=-0 \cdot 28, P<0 \cdot 00001)$. Nevertheless, these two components were not correlated but orthogonal, implying that the Western dietary pattern is not the opposite of the prudent dietary pattern.

\section{Discussion}

In the present study we found that OF use was an eating habit related to higher social class and healthier lifestyle and diet - all characteristics that predispose OF users to lower risks of chronic diseases that may affect fetal health during pregnancy. Thus, the study illustrates the major confounder problem that faces researchers who are seeking to tease out the relationship between OF consumption and health outcomes.

Very few studies have compared the diet of nonusers and frequent $\mathrm{OF}$ users and in general they are of poor quality. However, there seems to be an overall tendency towards higher intakes of fruit and vegetables and a lower intake of meat among OF users ${ }^{(42-44)}$, 
Table 4 Associations between nutrients from the diet (energy-adjusted) and organic consumption: pregnant women ( $n$ 60773), Danish National Birth Cohort (DNBC), 1996-2002

\begin{tabular}{|c|c|c|c|c|c|}
\hline \multirow[b]{3}{*}{ Nutrient } & \multirow{2}{*}{\multicolumn{2}{|c|}{ Non-users }} & \multicolumn{3}{|c|}{ Frequent users* } \\
\hline & & & \multicolumn{3}{|c|}{ Increase in intaket } \\
\hline & Mean & SD & Per d & $95 \% \mathrm{Cl}$ & $\%$ \\
\hline Energy (kJ) & 10058 & 2756 & 442 & 327,557 & 4 \\
\hline Fat $(\mathrm{g})$ & $83 \cdot 3$ & $16 \cdot 5$ & -4.9 & $-5 \cdot 5,-4 \cdot 2$ & -6 \\
\hline SFA (g) & $34 \cdot 9$ & $9 \cdot 0$ & $-2 \cdot 9$ & $-3 \cdot 3,-2 \cdot 6$ & -8 \\
\hline MUFA (g) & $26 \cdot 2$ & $5 \cdot 7$ & -1.5 & $-1 \cdot 7,-1 \cdot 2$ & -6 \\
\hline PUFA (g) & $11 \cdot 8$ & $2 \cdot 3$ & 0.09 & $-0.01,0.2$ & 1 \\
\hline$n-3(g)$ & $0 \cdot 7$ & $0 \cdot 2$ & 0.07 & $0.06,0.08$ & 11 \\
\hline$n-6(\mathrm{~g})$ & $2 \cdot 7$ & $0 \cdot 7$ & $-0 \cdot 19$ & $-0.22,-0.16$ & -7 \\
\hline Trans fatty acids (g) & $1 \cdot 6$ & $0 \cdot 7$ & $-0 \cdot 12$ & $-0.15,-0.09$ & -7 \\
\hline Cholesterol (mg) & 326 & $96 \cdot 7$ & $-10 \cdot 5$ & $-14 \cdot 5,-6 \cdot 4$ & -3 \\
\hline Protein $(\mathrm{g})$ & $87 \cdot 0$ & $14 \cdot 0$ & $2 \cdot 8$ & $2 \cdot 2,3 \cdot 4$ & 3 \\
\hline Carbohydrate (g) & 312 & $36 \cdot 9$ & $9 \cdot 1$ & $7 \cdot 5,10 \cdot 6$ & 3 \\
\hline Starch (g) & 107 & $31 \cdot 2$ & $1 \cdot 0$ & $-0 \cdot 3,2 \cdot 3$ & 1 \\
\hline Sugar (g) & 118 & $37 \cdot 8$ & $2 \cdot 0$ & $0.5,3.6$ & 2 \\
\hline Fibre $(\mathrm{g})$ & $24 \cdot 7$ & $6 \cdot 8$ & $3 \cdot 1$ & $2 \cdot 8,3 \cdot 4$ & 13 \\
\hline \multicolumn{6}{|l|}{ Vitamins } \\
\hline Vitamin A (RE) & 889 & 392 & $45 \cdot 6$ & $25 \cdot 6,62 \cdot 6$ & 5 \\
\hline Retinol $(\mu \mathrm{g})$ & 687 & 336 & $-77 \cdot 3$ & $-90 \cdot 7,-63.9$ & -11 \\
\hline$\beta$-Carotene $(\mu \mathrm{g})$ & 2338 & 2666 & 1465 & 1325,1604 & 63 \\
\hline Vitamin D $(\mu \mathrm{g})$ & $3 \cdot 0$ & $1 \cdot 6$ & 0.67 & $0.58,0.75$ & 22 \\
\hline Vitamin $E(\alpha-T E)$ & $7 \cdot 6$ & $1 \cdot 9$ & 0.56 & $0.48,0.64$ & 7 \\
\hline Vitamin $\mathrm{K}(\mu \mathrm{g})$ & $79 \cdot 9$ & $42 \cdot 4$ & $31 \cdot 4$ & $29 \cdot 3,33 \cdot 6$ & 39 \\
\hline Vitamin $B_{6}(\mathrm{mg})$ & $1 \cdot 5$ & 0.3 & $0 \cdot 14$ & $0 \cdot 13,0 \cdot 15$ & 9 \\
\hline Vitamin $B_{12}(\mu \mathrm{g})$ & $6 \cdot 2$ & $2 \cdot 3$ & $0 \cdot 27$ & $0.18,0.37$ & 4 \\
\hline Folate $(\mu \mathrm{g})$ & 330 & $65 \cdot 7$ & $36 \cdot 9$ & $33 \cdot 9,39 \cdot 8$ & 11 \\
\hline Vitamin C (mg) & 123 & $77 \cdot 9$ & $23 \cdot 8$ & $20 \cdot 4,27 \cdot 2$ & 19 \\
\hline \multicolumn{6}{|l|}{ Minerals } \\
\hline $\mathrm{Ca}(\mathrm{mg})$ & 1347 & 453 & $69 \cdot 4$ & $49 \cdot 9,88 \cdot 9$ & 5 \\
\hline $\mathrm{Mg}(\mathrm{mg})$ & 375 & $66 \cdot 1$ & $29 \cdot 6$ & $26 \cdot 8,32 \cdot 4$ & 8 \\
\hline $\mathrm{Fe}(\mathrm{mg})$ & $10 \cdot 9$ & $1 \cdot 6$ & 0.63 & $0.56,0.70$ & 6 \\
\hline $\mathrm{Zn}(\mathrm{mg})$ & $12 \cdot 2$ & $1 \cdot 9$ & $0 \cdot 33$ & $0.25,0.41$ & 3 \\
\hline Iodine $(\mu \mathrm{g})$ & 260 & $81 \cdot 9$ & $27 \cdot 2$ & $23 \cdot 4,31 \cdot 0$ & 10 \\
\hline Se $(\mu \mathrm{g})$ & $41 \cdot 2$ & $9 \cdot 5$ & $3 \cdot 6$ & $3 \cdot 2,4 \cdot 0$ & 9 \\
\hline $\mathrm{Cu}(\mathrm{mg})$ & $4 \cdot 5$ & $1 \cdot 8$ & 0.37 & $0.30,0.45$ & 8 \\
\hline
\end{tabular}

RE, retinol equivalents; $\alpha$-TE, $\alpha$-tocopherol equivalents.

${ }^{*}$ Change compared with non-users.

tMultivariate linear regression. Covariates: cohabitation status, age, smoking habits, parity, pre-pregnant BMI, occupational status, physical activity, urbanization and living area.

consistent with our findings. It has been argued that the healthier diet observed among frequent OF users can be explained by differences in food supply and prices ${ }^{(45)}$. Frequent users have a higher propensity to purchase OF products from speciality shops, but also from direct sales channels such as farm gates, box schemes, street stalls in urban areas, etc. that may affect OF product availability and consumption ${ }^{(46)}$. In the beginning of 2000 the availability of organic foods in supermarkets and discount stores was lower than today ${ }^{(47)}$, especially for organic meat, which can explain the lower OF intake for that product category.

A higher number of vegetarians among frequent $\mathrm{OF}$ users might in turn explain the lower intake of meat among frequent OF users in our study. However, stratification by vegetarianism showed a significantly lower adjusted intake of total meat (data not shown) among non-vegetarian OF users compared with non-vegetarian non-users. This finding is supported by a recent survey among 515 Danish consumers which showed that the highest quartile in relation to organic preferences consumed $50 \%$ less meat than non-users ${ }^{(48)}$.

Several efforts have been made to describe OF consumers through descriptive, socio-economic and behavioural factors. However, comparisons between studies are complicated by different market conditions between countries and different study methods. In general, reviews across countries show little consistency and no clear differences or patterns between organic and conventional food users ${ }^{(7,49)}$. Nevertheless, higher OF consumption tends to be related to vegetarianism ${ }^{(8,43,44,50,51)}$, educational level $^{(2,45,46)}$ and urbanization ${ }^{(2,45,46)}$ which is supported by our findings.

In previous studies frequent $\mathrm{OF}$ users have been described as 'intellectuals' from urban areas ${ }^{(2)}$ and the most common reason not to purchase OF products was lack of knowledge or awareness ${ }^{(7)}$. It has been hypothesized that higher educational level provides the consumer more information and experiences to believe that personal behaviour, including OF purchasing behaviour, and personal decisions 


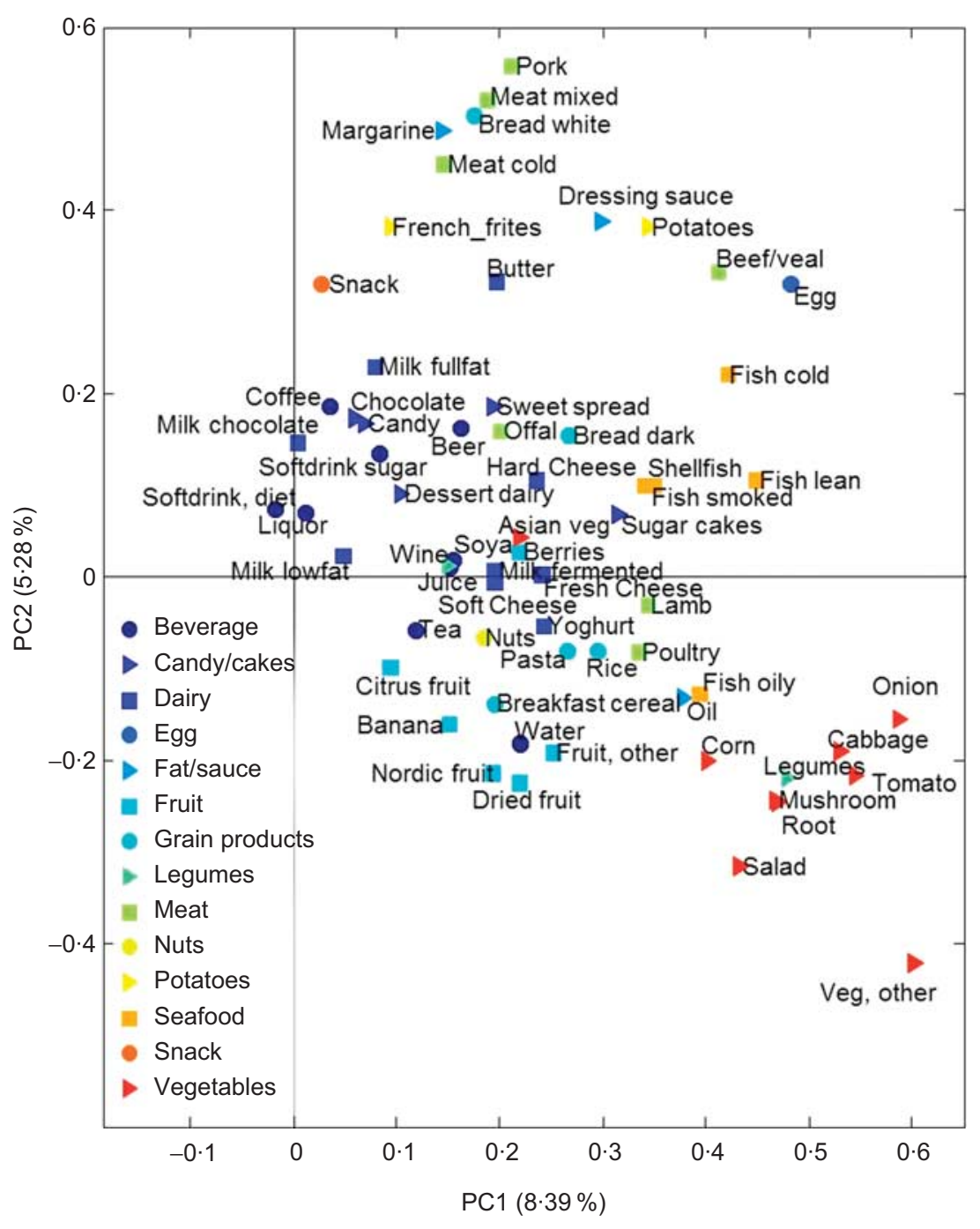

Fig. 1 Results from the principal components analysis: inter-correlation of food groups shown as a scatter plot of the first two principal components (PC1 and PC2) for the food groups. Food groups are coloured according to common food classes. Dietary data from pregnant women ( $n$ 60 773), Danish National Birth Cohort (DNBC), 1996-2002

affect other people ${ }^{(45)}$. We found occupational status to be strongly associated with OF preferences, which supports these previous studies. In relation to the higher OF use in or near the capital city our findings support the description of 'intellectuals' and the tendency to higher purchase in urban areas.

In general, income does not seem to explain differences in OF purchasing behaviour ${ }^{(7,49)}$ and income is a weak determinant in highly industrialized countries such as Denmark $^{(45)}$. Other findings suggest that OF users in some cases may have lower food expenditures than conventional households, despite the fact that OF products are more expensive, and this can be due to differences in dietary habits of the households ${ }^{(44)}$. Thus, higher price for OF products appears irrelevant in relation to other incentives underlying OF preferences.

There seems to be good agreement concerning incentives for OF use among countries ${ }^{(7,45)}$. Several studies have found that health considerations are one of the most important incentives for organic preferences followed by concern for the environment ${ }^{(2,7,12,49)}$ and concern about pesticide residues ${ }^{(10)}$. In fact, it has been argued that frequent $\mathrm{OF}$ users consider the concern for health and environment to be one and the same thing ${ }^{(45)}$. Since health apparently is a serious concern for OF users, it can be assumed that they follow recommendations about health and exercise to a higher extent than non-users. This is also reflected in our results, even after adjustment for occupational status.

The strength of our study is the large sample size as we have been able to include more than 60000 pregnant women. It can be argued that self-reported dietary intake may be prone to bias, such as over- or underestimation, but an FFQ is a valid method for classifying individuals according to high or low intake, which was the main interest with respect to OF consumption. The FFQ has 
been validated against a $7 \mathrm{~d}$ weighed food record and the validation showed that the FFQ was useful in separating high and low intake ${ }^{(29)}$. The dietary calculations were based on assumptions of average portions, sizes and standard recipes for complex dishes, which may have introduced bias in the estimates. In the present study we do not focus on the accuracy of specific nutrient estimates, but instead on the differences between estimates. Thus, we find the dietary intake between non-users and frequent users to be valid.

Until today, very few aetiological studies about organic consumption have been published. The major explanation underlying this may be found in the impact of surrounding multiple factors, unbalance in data, low compliance, and lack of knowledge about dietary components and the impact of pesticides on human health. Our findings add one more parameter because OF consumers in general seem to have a healthier lifestyle and diet. In relation to the previous findings of OF users being more conscious regarding health, observational studies aiming at examining the impact of OF use on human health are complicated.

It is relevant to consider whether OF consumption is part of a specific organic lifestyle including healthy diet, physical activity and health and environmental awareness. If this is the case it may be of no significance to estimate the relationship between OF use and health outcomes in observational studies, because the risk of chronic diseases already is lowered by the diet and exercise. However, it is still important to investigate whether OF products can contribute to lower risk of diseases. Therefore careful epidemiological modelling that can control for confounding factors is needed.

In theory, a randomized controlled trial would be the optimal study design for investigating health effects of human OF consumption. However, in many cases such a trial would require a long intervention period and strict control of foods consumed and would be affected by long study period, high costs and low compliance. Measurements of biomarkers in blood, e.g. pesticide residues and fatty acids composition, would be desirable; however, in a study including 60000 women this would be financially unfeasible. Moreover, possible health effects can be related to other factors that are undetectable in blood.

The DNBC gives us an opportunity to examine associations in observational studies; however, the statistical models used to analyse these associations must be designed to manage residual confounding and several covariates. Our approach is to devise a stratification strategy for selecting exchangeable groups of women for low and high OF consumption based on relevant confounders and our basis for this will be PCA. This will restrict the study population and hence reduce the statistical power, but in return produce conservative estimates with reduced bias for effects under the assumption of perfect exchangeability.

\section{Conclusions}

Frequent OF users in the DNBC had a healthier lifestyle and consumed a more prudent diet with higher intakes of fruit and vegetables, fibre, vitamins, minerals, $n-3$ fatty acids and less saturated fat. Furthermore, they had a higher occupational status and were living in urban areas, which together indicate an impact of a social gradient on OF purchasing behaviour. Our findings point to a major challenge in examining the impact of OF consumption on health in observational studies due to potentially irremediable confounding by generally healthier food choices among frequent users. Thus, in future studies it is crucial to manage this particular confounder problem. Our detailed analyses constitute a strong basis for such later advancement of strategies for analyses that can allow for unbalance in data, when we compare maternal organic and non-organic food consumers and their offspring in relation to health outcomes.

\section{Acknowledgements}

Sources of funding: The research was funded by the Danish Fund for Organic Agriculture and the European Union Integrated Research Project EARNEST (FOOD-CT-2005007036). The Centre for Fetal Programming is financed by the Danish Council for Strategic Research (grant no. 09-067124) and the DNBC has been financed by the March of Dimes Birth Defects Foundation, the Danish Heart Association, the Danish Medical Research Council, Sygekassernes Helsefond, the Danish National Research Foundation, the Danish Pharmaceutical Association, the Ministry of Health, the National Board of Health and Statens Serum Institut. Conflicts of interest: None of the authors had a personal or financial conflict of interest. Authors' contributions: S.B.P. and S.F.O.: study conception and design; S.B.P. and M.A.R.: analysis and interpretation of data; S.B.P. and M.A.R.: drafting of the manuscript, statistical analysis, full access to all of the data, responsibility for the integrity of the data and the accuracy of the data analysis; S.B.P., M.S., T.I.H. and S.F.O.: critical revision of the manuscript; S.B.P. and S.F.O.: acquisition of data and responsibility for the entire contents of the manuscript. Acknowledgements: The authors gratefully acknowledge Hanne Torjusen, Helle Margrete Meltzer, Anne Lise Brantsæeter and Margaretha Haugen from the Division of Environmental Medicine, Norwegian Institute of Public Health for inspiring cooperation, and the managerial team of the DNBC for their contributions.

\section{References}

1. Lea E (2005) Food, health, the environment and consumers' dietary choices. Nutr Diet 62, 21-25.

2. Torjusen H, Sangstad L, O'Doherty Jensen K et al. (2004) European Consumers' Conceptions of Organic Food: A Review 
of Available Research. Oslo: National Institute for Consumer Research.

3. Midmore P, Ayres N, Lund TB et al. (2008) Understanding the organic consumer through narratives: an international comparison. Presented at IFOAM Organic World Congress, Modena, Italy, 16-20 June 2008. http://orgprints.org/ 12574/ (accessed January 2011).

4. Wier M \& Andersen LM (2003) Consumer demand for organic foods - attitudes, values and purchasing behaviour. DARCOFenews $\mathbf{3}$, June issue.

5. Lund TB \& O'Doherty Jensen K (2008) Consumption of Organic Foods from a Life History Perspective: An Explorative Study among Danish Consumers. Copenhagen: Department of Human Nutrition, Sociology of Food Research Group, University of Copenhagen.

6. Ayres N \& Midmore P (2009) Consumption of Organic Foods from a Life History Perspective: An Exploratory Study of British Consumers. Aberystwyth: School of Management and Business, Aberystwyth University.

7. Yiridoe EK, Bonti-Ankomah S \& Martin RC (2005) Comparison of consumer perceptions and preference toward organic versus conventionally produced foods: a review and update of the literature. Renew Agric Food Syst 20, 193-205.

8. Schifferstein HNJ \& Oude Pphuis PAM (1998) Healthrelated determinants of organic food consumption in the Netherlands. Food Qual Prefer 9, 119-133.

9. Shepherd R, Magnusson M \& Sjoden PO (2005) Determinants of consumer behavior related to organic foods. Ambio 34, 352-359.

10. Byrne PJ, Bacon JR \& Toensmeyer UC (1994) Pesticide residue concerns and shopping location likelihood. Agribusiness 10, 491-501.

11. Huang CL (1996) Consumer preferences and attitudes towards organically grown produce. Eur Rev Agric Econ 23, 331-342.

12. Magnusson MK, Arvola A, Hursti UK et al. (2003) Choice of organic foods is related to perceived consequences for human health and to environmentally friendly behaviour. Appetite 40, 109-117.

13. Velimirov A, Huber M, Lauridsen C et al. (2009) Feeding trials in organic food quality and health research. J Sci Food Agric 90, 175-182.

14. Dangour AD, Dodhia SK, Hayter A et al. (2009) Nutritional quality of organic foods: a systematic review. Am J Clin Nutr 90, 680-685.

15. Williams CM (2002) Nutritional quality of organic food: shades of grey or shades of green? Proc Nutr Soc 61, 19-24.

16. Dangour AD, Lock K, Hayter A et al. (2010) Nutritionrelated health effects of organic foods: a systematic review. Am J Clin Nutr 92, 203-210.

17. Magkos F, Arvaniti F \& Zampelas A (2003) Organic food: nutritious food or food for thought? A review of the evidence. Int J Food Sci Nutr 54, 357-371.

18. Woese K, Lange D, Boess C et al. (1997) A comparison of organically and conventionally grown foods - results of a review of the relevant literature. J Sci Food Agric $\mathbf{7 4}$, 281-293.

19. Grinder-Pedersen L, Rasmussen SE, Bugel S et al. (2003) Effect of diets based on foods from conventional versus organic production on intake and excretion of flavonoids and markers of antioxidative defense in humans. $J$ Agric Food Chem 51, 5671-5676.

20. Kummeling I, Thijs C, Huber M et al. (2008) Consumption of organic foods and risk of atopic disease during the first 2 years of life in the Netherlands. Br J Nutr 99, 598-605.

21. Rist L, Mueller A, Barthel C et al. (2007) Influence of organic diet on the amount of conjugated linoleic acids in breast milk of lactating women in the Netherlands. Br J Nutr 97, 735-743.
22. Olsen J, Melbye M, Olsen SF et al. (2001) The Danish National Birth Cohort - its background, structure and aim. Scand J Public Health 29, 300-307.

23. Olsen SF, Mikkelsen TB, Knudsen VK et al. (2007) Data collected on maternal dietary exposures in the Danish National Birth Cohort. Paediatr Perinat Epidemiol 21, 76-86.

24. Willer H \& Kilcjer L (editors) (2011) The World of Organic Agriculture - Statistics and Emerging Trends 2011. Bonn and Frick: IFOAM and FiBL.

25. Overvad K, Tjonneland A, Haraldsdottir J et al. (1991) Development of a semiquantitative food frequency questionnaire to assess food, energy and nutrient intake in Denmark. Int J Epidemiol 20, 900-905.

26. National Food Institute, Technical University of Denmark (2009) Official Danish Food Composition Database. http:// www.foodcomp.dk (accessed November 2010).

27. Willett WC, Howe GR \& Kushi LH (1997) Adjustment for total energy intake in epidemiologic studies. Am J Clin Nutr 65, 4 Suppl., 1220S-1228S.

28. Friis S, Kruger Kjaer S, Stripp C et al. (1997) Reproducibility and relative validity of a self-administered semiquantitative food frequency questionnaire applied to younger women. J Clin Epidemiol 50, 303-311.

29. Mikkelsen TB, Osler M \& Olsen SF (2006) Validity of protein, retinol, folic acid and $n-3$ fatty acid intakes estimated from the food-frequency questionnaire used in the Danish National Birth Cohort. Public Health Nutr 9, 771-778.

30. Mikkelsen TB, Olsen SF, Rasmussen SE et al. (2007) Relative validity of fruit and vegetable intake estimated by the food frequency questionnaire used in the Danish National Birth Cohort. Scand J Public Health 35, 172-179.

31. Halldorsson TI, Thorsdottir I, Meltzer HM et al. (2009) Dioxin-like activity in plasma among Danish pregnant women: dietary predictors, birth weight and infant development. Environ Res 109, 22-28.

32. Halldorsson TI, Meltzer HM, Thorsdottir I et al. (2007) Is high consumption of fatty fish during pregnancy a risk factor for fetal growth retardation? A study of 44,824 Danish pregnant women. Am J Epidemiol 166, 687-696.

33. Halldorsson TI, Thorsdottir I, Meltzer HM et al. (2008) Linking exposure to polychlorinated biphenyls with fatty fish consumption and reduced fetal growth among Danish pregnant women: a cause for concern? Am J Epidemiol 168, 958-965.

34. Klemmensen AK, Tabor A, Østerdal ML et al. (2009) Intake of vitamin $\mathrm{C}$ and $\mathrm{E}$ in pregnancy and risk of pre-eclampsia: prospective study among 57,346 women. BJOG 116, 964-974.

35. Knudsen VK, Hansen HS, Ovesen L et al. (2007) Iron supplement use among Danish pregnant women. Public Health Nutr 10, 1104-1110.

36. Mikkelsen TB, Osler M, Orozova-Bekkevold I et al. (2006) Association between fruit and vegetable consumption and birth weight: a prospective study among 43,585 Danish women. Scand J Public Health 34, 616-622.

37. Mikkelsen TB, Osterdal ML, Knudsen VK et al. (2008) Association between a Mediterranean-type diet and risk of preterm birth among Danish women: a prospective cohort study. Acta Obstet Gynecol Scand 87, 325-330.

38. Oken E, Osterdal ML, Gillman MW et al. (2008) Associations of maternal fish intake during pregnancy and breastfeeding duration with attainment of developmental milestones in early childhood: a study from the Danish National Birth Cohort. Am J Clin Nutr 88, 789-796.

39. Osterdal ML, Strom M, Klemmensen AK et al. (2009) Does leisure time physical activity in early pregnancy protect against pre-eclampsia? Prospective cohort in Danish women. BJOG 116, 98-107.

40. Strom M, Mortensen EL, Halldorsson TI et al. (2009) Fish and long-chain $n$-3 polyunsaturated fatty acid intakes 
during pregnancy and risk of postpartum depression: a prospective study based on a large national birth cohort. Am J Clin Nutr 90, 149-155.

41. Wold S, Esbensen K \& Geladi P (1987) Principal component analysis. Chemometr Intell Lab 2, 37-52.

42. Denver S, Christensen T \& Krarup S (2007) Får økologiske forbrugere oftere 6 om dagen? (Do organic users consume more often 6 servings per day?) Tidsskrift for Landøkonomi 2, 109-118.

43. Holt G (1992) Investigating the diet of 'organic eaters'. Nutr Food Sci 6, 13-16.

44. Brombacher J \& Hamm U (1990) Expenses for nutrition with food from organic agriculture. Ecol Farm 1, 13-16.

45. O'Doherty Jensen K, Larsen HN, Mølgaard JP et al. (2001) Økologiske fødevarer og menneskets sundhed (Organic Foods and Human Health). FØJO-rapport nr. 14. Tjele: Forskningscenter for Økologisk Jordbrug (Centre for Organic Food Research, Denmark).

46. Wier M, O'Doherty Jensen K, Andersen LM et al. (2008) The character of demand in mature organic food markets:
Great Britain and Denmark compared. Food Policy 33, 406-421.

47. Statistics Denmark (2011) StatBank Denmark. http:// www.statbank.dk/statbank5a/default.asp?w=1920 (accessed January 2011).

48. FDB Analyse (2010) Økologiske forbrugere belaster klimaet mindre (Organic consumers affect the environment to a lesser extent). http://fdb.dk/nyhed/\%C3\%B8kologiskeforbrugere-belaster-klimaet-mindre (accessed February 2011).

49. Bonti-Ankomah S \& Yiridoe EK (2006) Organic and Conventional Food: a Literature Review of the Economics of Consumer Perceptions and Preferences - Final Report. Ottawa, ON and Truro, NS: Agriculture and Agri-Food Canada and Nova Scotia Agricultural College.

50. Torjusen H, Brantsaeter AL, Haugen M et al. (2010) Characteristics associated with organic food consumption during pregnancy; data from a large cohort of pregnant women in Norway. BMC Public Health 10, 775.

51. Onyango BM (2007) Purchasing organic food in US food systems - a study of attitudes and practice. Br Food J 109, 399-411. 